\title{
CTX-M-I5 Positive Escherichia coli and Klebsiella pneumoniae Outbreak in the Neonatal Intensive Care Unit of a Maternity Hospital in Ha'il, Saudi Arabia
}

\author{
Mohammed Almogbel ${ }^{1}$ \\ Ahmed Altheban ${ }^{2}$ \\ Mohammed Alenezi ${ }^{3}$ \\ Khalid Al-Motair' \\ Godfred A Menezes ${ }^{4}$ \\ Mohammed Elabbasy' \\ Sahar Hammam ${ }^{3}$ \\ John P Hays (D) $^{5}$ \\ Mushtaq A Khan (iD)
}

'Molecular Diagnostic and Personalized Therapeutics Unit, College of Applied Medical Sciences, University of Ha'il, Ha'il, Saudi Arabia; ${ }^{2}$ College of Nursing, University of Ha'il, Ha'il, Saudi Arabia; ${ }^{3}$ Maternity Hospital, Ha'il, Saudi Arabia; ${ }^{4}$ Department of Medical Microbiology and Immunology, RAK College of Medical Sciences, RAK Medical \& Health Sciences University, Ras Al Khaimah, United Arab Emirates; ${ }^{5}$ Department of Medical Microbiology and Infectious Diseases, Erasmus University Medical Centre (Erasmus MC), Rotterdam, The Netherlands; ${ }^{6}$ Department of Microbiology and Immunology, College of Medicine and Health Sciences, United Arab Emirates University, Al Ain, United Arab Emirates

\begin{abstract}
Objective: The aim of this study was to retrospectively characterize $E$. coli and $K$. pneumoniae isolates obtained from neonates during a suspected NICU outbreak of infection in Ha'il, Saudi Arabia during a period of one month (April 2014).

Methods: Antibiotic susceptibility patterns, molecular characterization for antibiotic-resistant genes (blaTEM, blaSHV, and blaCTX-M), and genotyping by PFGE and MLST were performed.

Results: A total of 24 E. coli and $48 \mathrm{~K}$. pneumoniae isolates were cultured from neonates that had been admitted to the NICU. Among E. coli, the majority of isolates (19/24) were ESBL-positive and all of these nineteen (100\%) harbored the CTX-M-15 gene. A total of $15 \%(3 / 19)$ were co-producers of CTX-M-15 and SHV-12, and $68.4 \%(13 / 19)$ were coproducers of CTX-M-15 and TEM-1. Among K. pneumoniae isolates, $87.5 \%$ (42/48) were ESBL positive with $92.85 \%$ (39/42) of these isolates containing the CTX-M-15 gene. A total of $97 \%$ (38/39) of $K$. pneumoniae were co-producers of CTX-M-15 and SHV-12, and $88 \%$ (37/42) were positive for TEM-1. Furthermore, 85.7\% (36/42) K. pneumoniae were coproducers of CTX-M-15 and TEM-1. The majority of E. coli isolates (18/19 isolates) were grouped into two genetic clusters by pulsed field gel electrophoresis (PFGE) and all the isolates were found to be ST-131 type. In contrast, K. pneumoniae (31/42) isolates belonged to a single genotypic lineage, and all $(100 \%)$ isolates belonged to the ST-14 type.

Conclusion: This is the first report of CTX-M-15-positive, ESBL E. coli, and K. pneumoniae isolates recovered from an outbreak in an NICU in Ha'il, Saudi Arabia. It is alarming to note the high rate of outbreak isolates with simultaneous production of CTX-M-15 and SHV12 conferring high-level resistance to oxyimino-cephalosporins.
\end{abstract}

Keywords: extended spectrum $\beta$-lactamases, NICU outbreak, CTX-M-15, E. coli, K. pneumoniae

\section{Introduction}

Neonates are at a very high risk of developing life-threatening bacterial infections due to their under-developed immune systems, resulting in significant morbidity and mortality. ${ }^{1}$ The problem among these neonates in ICU settings is further complicated due to the use of broad-spectrum antibiotics, contact with healthcare workers (HCWs), and exposure to invasive and surgical procedures. ${ }^{2}$ In outbreak settings, NICU patients are particularly vulnerable to colonization and infection with pathogens such as multidrug-resistant
Correspondence: Mushtaq A Khan Department of Microbiology and Immunology, College of Medicine and Health Sciences, United Arab Emirates University, Al Ain, United Arab Emirates $\mathrm{Tel}+971555368100$

Email mushtaq.khan@uaeu.ac.ae 
Gram-negative bacteria. ${ }^{3}$ In the majority of cases, these healthcare-associated infections are caused by Escherichia coli and Klebsiella pneumoniae and require the administration of multiple antibiotics. E. coli is a common cause of community and healthcare-associated diseases, and over the past few decades, E. coli strains isolated from community-acquired infections have become increasingly resistant to antibiotics. ${ }^{4,5}$ Further, bloodstream infections caused by $K$. pneumoniae are also often reported in neonatal intensive care units (NICUs). ${ }^{6,7}$ Additionally, there has been rapid and global dissemination of extended-spectrum- $\beta$-lactamase (ESBL) producing $E$. coli and $K$. pneumoniae in hospital settings, which complicates and limits current antibiotic treatment options. ${ }^{8}$ This increase has been mainly due to the successful dissemination of CTX-M15 gene-carrying mobile genetic elements. ${ }^{9,10}$ CTX-M-15, the most widely distributed of these enzymes, was first found in isolates of Enterobacteriaceae from India, but is now prevalent almost everywhere in the world. It appears to be associated with epidemic plasmids flanked with insertion sequences that facilitate easy spread and hyper production of $\beta$-lactamase, which may, in part, explain the gene's rapid spread. ${ }^{11}$

ESBL-producing E. coli and K. pneumoniae have previously been reported to cause outbreaks of infection in neonatal intensive care units (NICUs). ${ }^{11,12}$ However, to our knowledge, this is the first report of a CTX-M-15-positive $E$. coli and $K$. pneumoniae outbreak in the neonatal intensive care unit of a maternity hospital in Saudi Arabia. In this study, we retrospectively characterized 19-ESBL-positive E. coli, and 42-ESBL-positive $K$. pneumoniae isolates obtained from an outbreak of infection at a neonatal intensive care unit (NICU) in the Ha'il region of Saudi Arabia.

\section{Materials and Methods}

\section{Sample Collection}

During April 2014, an outbreak of 3rd generation cephalosporin resistant bacterial infection was suspected within a neonatal intensive care unit in a maternity hospital at Ha'il, Saudi Arabia. As part of the investigations into the outbreak during a one-month period (April 2014), a total of 821 samples were screened, including 407 patient and 414 other samples (comprising healthcare workers, staff and swabs from the NICU environment). Extended-spectrum $\beta$-lactamase positive bacterial strains of E. coli and K. pneumoniae cases were defined as those patients admitted to the NICU from whom at least one sample recovered during the NICU stay contained an ESBL producer. Only one non-repetitive

Table I Specific PCR Primer Pairs Used in This Study.

\begin{tabular}{|c|c|c|}
\hline Resistance Type & Primer Name & Primer Sequence (5'- 3') \\
\hline blaTEM & $\begin{array}{l}\text { TEMFU } \\
\text { TEMRU } \\
\text { TEMFLF } \\
\text { TEMFLR }\end{array}$ & $\begin{array}{l}\text { TCGTGTCGCCCTTATTCCCTTTTT } \\
\text { GCGGTTAGCTCCTCCGGTCCTC } \\
\text { GAAGACGAAAGGGCCTCGTG } \\
\text { GGTCTGACAGTTACCAATGC }\end{array}$ \\
\hline blaSHV & $\begin{array}{l}\text { SHVFU } \\
\text { SHVRU } \\
\text { SHVFLF } \\
\text { SHVFLR }\end{array}$ & $\begin{array}{l}\text { GTGGATGCCGGTGACGAACAGC } \\
\text { TGGCGCAAAAAGGCAGTCAATCCT } \\
\text { CGCCGGGTTATTCTTATTTGTCGC } \\
\text { TCTTTCCGATGCCGCCGCCAGTCA }\end{array}$ \\
\hline blaCTX-M & $\begin{array}{l}\text { CTX-MUF } \\
\text { CTX-MUR } \\
\text { CTX-M gRpIF } \\
\text { CTX-M gRpIR } \\
\text { CTX-M gRp2F } \\
\text { CTX-M gRp2R } \\
\text { CTX-M gRp9F } \\
\text { CTX-M gRp9R } \\
\text { CTX-M gRp8F } \\
\text { CTX-M gRp8/25R } \\
\text { CTX-M gRp25F } \\
\text { CTXMSeqF } \\
\text { CTXMSeqR }\end{array}$ & $\begin{array}{l}\text { CGCTTTGCGATGTGCAG } \\
\text { ACCGCGATATCGTTGGT } \\
\text { AAAAATCACTGCGCCAGTTC } \\
\text { AGCTTATTCATCGCCACGTT } \\
\text { CGACGCTACCCCTGCTATT } \\
\text { CCAGCGTCAGATTTTTCAGG } \\
\text { CAAAGAGAGTGCAACGGATG } \\
\text { ATTGGAAAGCGTTCATCACC } \\
\text { TCGCGTTAAGCGGATGATGC } \\
\text { AACCCACGATGTGGGTAGC } \\
\text { GCACGATGACATTCGGG } \\
\text { GTTCGTCTCTTCCAGAATAAGG } \\
\text { CAGCACTTTTGCCGTCTAAG }\end{array}$ \\
\hline
\end{tabular}

Note: Adapted from Hassan H, Abdalhamid B. Molecular characterization of extended-spectrum beta-lactamase producing Enterobacteriaceae in a Saudi Arabian tertiary hospital. J Infect Dev Ctries. 20I4;8(3):282-288. doi:10.3855/jidc.3809. ${ }^{14}$ 
ESBL-positive bacterial isolate was processed from each ESBL- positive patient.

\section{Bacterial Identification and Antibiotic Susceptibility Testing}

The cultured bacterial isolates were identified by a routine culture-based identification system, as well as MALDITOF mass spectrometry (Bruker Daltonics-Germany). ESBL's were initially screened for reduced susceptibility to cefpodoxime, cefotaxime, ceftriaxone, ceftazidime, or aztreonam, and then by performing phenotypic confirmatory test by demonstrating a synergistic effect between an indicator cephalosporin and a $\beta$-lactamase inhibitor. The ceftazidime $(30 \mu \mathrm{g})$ discs alone and in combination with clavulanic acid (ceftazidime + clavulanic acid, 30/10 $\mu \mathrm{g}$ discs) were applied onto a plate of Mueller Hinton Agar (MHA), which was inoculated with the test strain. An increase of $\geq 5 \mathrm{~mm}$ in the zone of inhibition of the combination discs in comparison to the ceftazidime disc alone was considered to be a marker for ESBL production. ${ }^{13}$ Additional antibiotic susceptibility profiling of the positive isolates was then performed using the MicroScan WalkAway-40 plus (Indianapolis, United States) system.

\section{DNA Extraction}

Bacterial DNA was as isolated using the automated QIAcube device and the QIAamp DNA Mini Kit (Qiagen, Hilden, Germany) according to the manufacturer's instructions.

\section{Identification of $\beta$-Lactamase Genes}

Genes encoding for $\beta$-lactamase enzymes (blaCTX-M, blaSHV, and blaTEM) were detected using PCR and sequencing according to a previously published method. ${ }^{14}$ The specific primer pairs that are used in the current study are shown in Table 1.

\section{Pulsed Field Gel Electrophoresis (PFGE) and Multilocus Sequence Typing (MLST)}

PFGE was performed using $X b a \mathrm{I}$-digested fragments of bacterial chromosomal DNA, with fragment separation achieved in $0.8 \%$ agarose. Electrophoresis conditions comprised a constant voltage of $6 \mathrm{~V} / \mathrm{cm}$ at $148^{\circ} \mathrm{C}$ and pulse times of 3.5-25 s increased linearly over $12 \mathrm{~h}$ (block 1 ), followed by $1-5 \mathrm{~s}$ increased over an 8-hour period. Gel patterns were analyzed using BioNumerics software (Applied Maths) with the band tolerance set at $1.0 \%{ }^{15}$
Multilocus sequence typing was performed using seven housekeeping genes in E. coli and K. pneumoniae. ${ }^{16,17}$

\section{Results}

\section{Prevalence and Susceptibility Patterns}

A total of $24 \mathrm{E}$. coli isolates were cultured from neonates, with the majority of isolates (19/24) being ESBL-positive (Figure 1) and resistant to 3rd generation cephalosporins. However, all of the isolates were susceptible to aminoglycosides (amikacin, gentamicin and tobramycin), except for 2 isolates that were intermediate resistant to tobramycin. All isolates were also susceptible to cefoxitin, ciprofloxacin, levofloxacin and imipenem. Additionally, 48 K. pneumoniae isolates were also cultured from neonates, with the majority of isolates $87.5 \%$ (42/48) being ESBL positive (Figure 2) and resistant to 3rd generation cephalosporins. Overall 90\% (37/ 42) of these ESBL-positive $K$. pneumoniae isolates were resistant to gentamicin and tobramycin but all $42(100 \%)$ isolates were susceptible to amikacin. However, all of the $K$. pneumoniae isolates were susceptible to ciprofloxacin, levofloxacin and imipenem. None of the umbilical isolates from neonates (E. coli or $K$. pneumoniae) were cultured from neonates with omphalitis (inflammation of the umbilical cord stump).

\section{Characterization of $\beta$-Lactamase Genes}

Characterization of $\beta$-lactamase genes among $E$. coli isolates revealed that $100 \%(19 / 19)$ of ESBL-positive E. coli isolates were found to harbor the CTX-M-15 gene. Further, 15\% (3/19) of E. coli isolates possessed both CTX-M-15 and SHV-12 genes, with 68.4\% (13/19) being TEM-1 positive (Figure 1). Among $K$. pneumoniae, 92.85\% (39/42) of isolates contained the CTX-M-15 gene. A total of $95 \%$ (38/40) of K. pneumoniae possessed both the CTX-M-15 and SHV-12 genes. Further, 88\% (38/ 42) were positive for TEM-1 (Figure 2).

\section{PFGE and MLST Genotyping}

PFGE result showed that the majority of $E$. coli isolates grouped into 2 genetic clusters at $80 \%$ similarity (18/19 isolates) and 12 genotypes at $95 \%$ similarity. The majority (31/42) of K. pneumoniae isolates belonged to a single genotypic lineage at the $85 \%$ similarity level and 16 genotypes at the $95 \%$ similarity level.

MLST results showed that all 19 E. coli isolates belonged to ST131 and all $K$. pneumoniae isolates belonged to ST 14 . 


\begin{tabular}{|c|c|c|c|c|c|c|c|c|c|c|c|c|c|c|c|}
\hline$\stackrel{8}{\perp}, \ldots, \ldots, \ldots$ & ID & SOURCE & AK & GM & то & AMP & CFT & CAZ & CIP & LEVO & IMP & MLST & TEM & SHV & стХ-M \\
\hline & MB1501644 & RECTAL & $\mathrm{s}$ & $\mathrm{s}$ & $s$ & $\mathrm{R}$ & $\mathrm{R}$ & $\mathrm{R}$ & $\mathrm{s}$ & $\mathrm{s}$ & $\mathrm{s}$ & 131 & $\mathrm{~N}$ & $\mathrm{~N}$ & 15 \\
\hline & MB1501651 & UMBILICAL & $s$ & $\mathrm{~s}$ & I & $\mathrm{R}$ & $\mathrm{R}$ & $\mathrm{R}$ & $s$ & $\mathrm{~s}$ & $\mathrm{~s}$ & 131 & $\mathrm{~N}$ & $\mathrm{~N}$ & 15 \\
\hline & MB1501628 & RECTAL & s & $s$ & s & $\mathrm{R}$ & $\mathrm{R}$ & $\mathrm{R}$ & $s$ & $s$ & s & 131 & 1 & 2 & 15 \\
\hline & MB1501665 & RECTAL & $\mathrm{s}$ & $s$ & $s$ & $\mathrm{R}$ & $\mathrm{R}$ & $\mathrm{R}$ & $s$ & $s$ & $\mathrm{~s}$ & 131 & 1 & $\mathrm{~N}$ & 15 \\
\hline & MB1501607 & RECTAL & $\mathrm{s}$ & $\mathrm{s}$ & $\mathrm{s}$ & $\mathrm{R}$ & $\mathrm{R}$ & $\mathrm{R}$ & $s$ & $\mathrm{~s}$ & $s$ & 131 & 1 & 12 & 15 \\
\hline & MB1501636 & RECTAL & $s$ & $s$ & 1 & $\mathrm{R}$ & $\mathrm{R}$ & $\mathrm{R}$ & s & $s$ & $\mathrm{~s}$ & 131 & $\mathrm{~N}$ & $\mathrm{~N}$ & 15 \\
\hline & MB1501612 & RECTAL & $s$ & $s$ & s & $\mathrm{R}$ & $\mathrm{R}$ & $\mathrm{R}$ & $s$ & s & $s$ & 131 & $\mathrm{~N}$ & $\mathrm{~N}$ & 15 \\
\hline & MB1501661 & RECTAL & $s$ & $\mathrm{~s}$ & $\mathrm{~s}$ & $s$ & $\mathrm{R}$ & $\mathrm{s}$ & $\mathrm{s}$ & $\mathrm{s}$ & $\mathrm{s}$ & 131 & $\mathrm{~N}$ & 2 & 15 \\
\hline & MB1501658 & NOSE & $\mathrm{s}$ & $\mathrm{s}$ & $\mathrm{s}$ & $\mathrm{R}$ & $\mathrm{R}$ & $\mathrm{R}$ & $s$ & s & $s$ & 131 & $\mathrm{~N}$ & 2 & 15 \\
\hline & MB1501659 & RIGHT HAND & s & $s$ & $s$ & $\mathrm{R}$ & $\mathrm{R}$ & $\mathrm{R}$ & $s$ & $\mathrm{~s}$ & $\mathrm{~s}$ & 131 & 1 & 2 & 15 \\
\hline & MB1501652 & DRESSING & $\mathrm{s}$ & $s$ & $s$ & $\mathrm{R}$ & $\mathrm{R}$ & $\mathrm{R}$ & $s$ & $\mathrm{~s}$ & $s$ & 131 & 1 & 2 & 15 \\
\hline & MB1501666 & RIGHT HAND & s & $\mathrm{s}$ & $\mathrm{s}$ & $\mathrm{R}$ & $\mathrm{R}$ & $\mathrm{R}$ & $s$ & $\mathrm{~s}$ & $s$ & 131 & 1 & 2 & 15 \\
\hline & MB1501620 & UMBILICAL & $s$ & $s$ & s & $\mathrm{R}$ & $\mathrm{R}$ & $\mathrm{R}$ & $s$ & $\mathrm{~s}$ & $s$ & 131 & 1 & 12 & 15 \\
\hline & MB1501615 & RECTAL & $\mathrm{s}$ & $\mathrm{s}$ & $s$ & $\mathrm{R}$ & $\mathrm{R}$ & $\mathrm{R}$ & $s$ & $\mathrm{~s}$ & $s$ & 131 & 1 & 2 & 15 \\
\hline & MB1501616 & NOSE & $s$ & $s$ & $s$ & $R$ & $\mathrm{R}$ & $\mathrm{R}$ & $s$ & $s$ & $s$ & 131 & 1 & N & 15 \\
\hline & MB1501632 & RECTAL & $s$ & $s$ & s & $\mathrm{R}$ & $\mathrm{R}$ & $\mathrm{R}$ & $s$ & $s$ & $s$ & 131 & 1 & 2 & 15 \\
\hline L & MB1501630 & RECTAL & $s$ & $s$ & $s$ & $\mathrm{R}$ & $\mathrm{R}$ & $\mathrm{R}$ & $s$ & $s$ & $\mathrm{~s}$ & 131 & 1 & 2 & 15 \\
\hline & MB1501624 & RECTAL & $s$ & $s$ & $\mathrm{~s}$ & $\mathrm{R}$ & $\mathrm{R}$ & $\mathrm{R}$ & $s$ & $s$ & $\mathrm{~s}$ & 131 & 1 & $\mathrm{~N}$ & 15 \\
\hline & MB1501631 & NOSE & S & $s$ & $S$ & $\mathrm{R}$ & $R$ & $\mathrm{R}$ & $s$ & S & $S$ & 131 & 1 & 12 & 15 \\
\hline
\end{tabular}

Figure I PFGE patterns from 19 ESBL-positive E. coli isolates obtained from an NICU outbreak of infection from Ha'il, Saudi Arabia in 20I4. Cluster analysis was performed using the method of DICE with UPGMA with band tolerances set to $1.0 \%$. MIC values $(\mu \mathrm{g} / \mathrm{mL})$ : amikacin $(A K) S=\leq I 6$; gentamicin $(\mathrm{GM}) \mathrm{S}=\leq 4$; tobramycin $(\mathrm{TOB}) \mathrm{S} \leq 4, \mathrm{I}=$ 8; ampicillin (AMP) $S \leq 8, R \geq 32$; cefoxitin (CFT) $R \geq 32$; ceftazidime (CAZ) $S \leq 4, R \geq 16$; ciprofloxacin (CIP) $S \leq 0.25$; levofloxacin (LEVO) $S \leq 0.5$, imipenem (IMI) $S \leq I$. Abbreviations: MLST, I3I - Multilocus sequence type I3I. TEM, N - no TEM gene detected; I - TEM I detected. SHV, N - no SHV gene detected; 2 - SHV 2 detected; 12 SHV 12 detected. CTX-M, 15 - CTX-M-15 detected.

\section{Discussion}

Our results indicate some interesting features of retrospective E. coli and $K$. pneumoniae isolates associated with NICU infections in Saudi Arabia. For example, a 2year study of a neonatal intensive care unit in Riyadh, Saudi Arabia in 2000 already indicated that E. coli was the most common infecting organism associated with early onset sepsis. ${ }^{18}$ Additionally, E. coli and K. pneumoniae have been reported as the most commonly isolated bacteria associated with neonatal septicemia in Saudi Arabia (Riyadh) at least as far back as the 1980's. ${ }^{19}$

Within Saudi Arabia, the dominance of ST131 E. coli isolates in population-based studies has previously been reported, extending back to at least 2011. Further, this clonal lineage of $E$. coli continues to be problematic in Saudi Arabia, ${ }^{20,21}$ although infections associated with the ST131 clone are not only isolated to Saudi Arabia. ${ }^{22}$ Additionally, associations between E. coli ST131 clonal type and carriage of the CTX-M-15 gene on IncF[F2:A1: B-] (Group 2) plasmids of Clade 2 isolates has been reported. ${ }^{23}$ CTX-M-15 positive E. coli and K. pneumoniae have been reported to cause outbreaks in neonatal intensive care units (NICUs) worldwide. ${ }^{9-12}$ Additionally, $K$. pneumoniae ST14 isolates expressing CTX-M-15 have been reported as being associated with neonatal sepsis in Tanzania between 2009 and 2010, as well as in Spain in $2008^{24,25}$

In Saudi Arabia, several studies have reported the dominance of CTX-M-15 genes among Enterobacteriaceae, ${ }^{26,27}$ including in K. pneumoniae isolates associated with an NICU in 2007. ${ }^{28}$ In fact, among CTX-M genes, CTX-M15 is the most prevalent reported worldwide. ${ }^{29} \mathrm{CTX}-\mathrm{M}-15$ positive Enterobacteriaceae isolates from Riyadh in 2009 were associated with a high prevalence of quinolone resistance, although our isolates were all quinolone susceptible.27 This may reflect differences in antibiotic prescribing practices between different cities within Saudi Arabia. The threat posed by multi-drug resistant $K$. pneumoniae to the Arabian gulf countries was recently highlighted by Bindayna, Joji, Ezzat and Jahrami ${ }^{30}$ in a meta-analysis of 28 published articles reported a high prevalence of $b l a_{\text {OXA- }}$ 


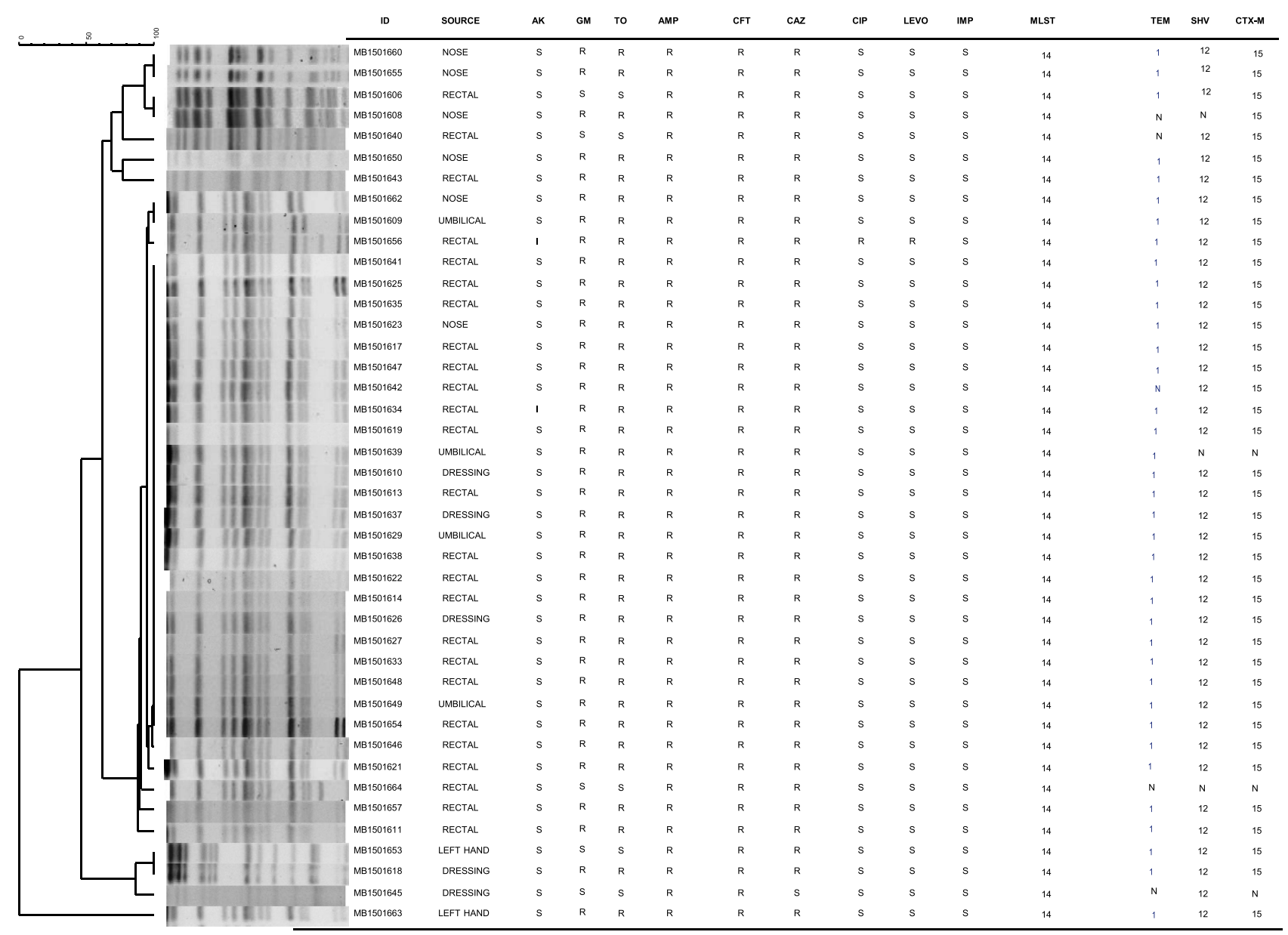

Figure 2 PFGE patterns from 42 ESBL-positive K. pneumoniae isolates obtained from an NICU outbreak of infection from Ha'il, Saudi Arabia in 20I4. Cluster analysis was performed using the method of DICE with UPGMA with band tolerances set to $1.0 \%$. MIC values $(\mu \mathrm{g} / \mathrm{mL})$ : amikacin $(A K) S \leq I 6, I=32$; gentamicin $(\mathrm{GM}) \mathrm{S} \leq 4, \mathrm{R}$ $\geq 16$; tobramycin (TOB) $S \leq 4, I=8, R \geq 16$; ampicillin (AMP) $R \geq 32$; cefoxitin (CFT) $R \geq 32$; ceftazidime (CAZ) $S \leq 4$, $R \geq I 6$; ciprofloxacin (CIP) $S \leq 0.25$, $R \geq I$; levofloxacin (LEVO) $S \leq 0.5, R \geq 2$; imipenem (IMI) $S \leq I$.

Abbreviations: MLST, 14 - Multilocus sequence type 14. TEM, N - no TEM gene detected; I - TEM I detected. SHV, N - no SHV gene detected; 12 - SHV I2 detected. CTX-M, N - no CTX-M gene detected; 15 - CTX-M-15 detected.

48 and bla $_{\mathrm{CTX}-\mathrm{M}}$, followed by $b l a_{\mathrm{SHV}}$, bla $_{\mathrm{TEM}}, b l a_{\mathrm{NDM}-1}$ and $b l a_{\mathrm{VIM}}$ genes in K. pneumoniae. Unfortunately, the consequences of such multi-drug resistance to citizens of Saudi Arabia are not a recent phenomenon, as indicated by the current publication. In 2019, a 33-month surveillance study in three pediatric and neonatal intensive care units in Riyadh concluded that aminoglycosides represented $45.4 \%$ of monitored antimicrobials used in neonatal ICU followed by cephalosporins (30.4\%). Prescribing practices may vary between hospitals, but this information could indicate why combined aminoglycoside and cephalosporin (K. pneumoniae) and cephalosporin (E. coli) antimicrobial resistance is extensively observed in our results. ${ }^{31}$ Mechanical ventilation and total parenteral nutrition were previously identified as significant risk factors for nosocomial infection within a neonatal care unit in South-Western
Saudi Arabia. ${ }^{32}$ Other risk factors associated with neonatal sepsis from Riyadh (2011-2015) were prematurity, as well as multiparty and delivery by caesarean section. Similar to the current publication this same report indicated that all Gram-negative organisms isolated were sensitive to amikacin. ${ }^{33}$ It has previously been reported that the carriage of the bla SHV -related genes may be universal in K. pneumoniae isolates, ${ }^{34}$ while three $K$. pneumoniae isolates from our collection were repeatedly SHV negative using PCR. Various technical issues (DNA extraction, PCR thermocycling, etc.) or biological factors (geographical location, nucleotide changes in primer binding sequences, etc.) could have been responsible for this discrepancy. However, further investigations are beyond the scope of this publication and the negative results do not influence the authors' conclusion. 


\section{Conclusions}

This is the first report of CTX-M-15-positive, ESBL E. coli and $K$. pneumoniae isolates recovered from an outbreak in an NICU in Ha'il, Saudi Arabia. It is alarming to note the high rate of outbreak isolates with simultaneous production of CTX-M-15 and SHV-12 conferring high-level resistance to oxyimino-cephalosporins, even in isolates cultured in 2014. Further studies are required in order to indicate the extent and spread of CTX-M-15-positive Enterobacteriaceae in more recent years within Saudi Arabia.

\section{Ethical Approval}

There was no approval required for this research by an institutional review board or ethics committee.

\section{Funding}

This study was funded by the King Abdul-Aziz City of Science and Technology (KACST) through project reference 153-34 and UAE University start up grant through project reference $12 \mathrm{M} 003$.

\section{Disclosure}

The authors report no conflicts of interest in this work.

\section{References}

1. Naher HS, Al-Sa'ady AT. Review on bacterial etiology of neonatal infections. Eurasia J Biosci. 2020;14:7619-7630.

2. Advani SD, Murray TS, Murdzek CM, Aniskiewicz MJ, Bizzarro MJ. Shifting focus toward healthcare-associated bloodstream infections: the need for neonatal intensive care unit-specific NHSN definitions. Infect Control Hosp Epidemiol. 2020;41(2):181-186. doi:10.1017/ ice. 2019.310

3. Flannery DD, Akinboyo IC, Mukhopadhyay S, et al. Antibiotic Susceptibility of Escherichia coli Among Infants Admitted to Neonatal Intensive Care Units Across the US From 2009 to 2017. JAMA Pediatr. 2021;175(2):168-175. doi:10.1001/ jamapediatrics.2020.4719

4. Bonten M, Johnson JR, van den Biggelaar AHJ, et al. Epidemiology of Escherichia coli Bacteremia: a Systematic Literature Review. Clin Infect Dis. 2020;72(7):1211-1219. doi:10.1093/cid/ciaa210

5. Larramendy S, Deglaire V, Dusollier P, et al. Risk Factors of Extended-Spectrum Beta-Lactamases-Producing Escherichia coli Community Acquired Urinary Tract Infections: a Systematic Review. Infect Drug Resist. 2020;13:3945-3955. doi:10.2147/IDR.S269033

6. Hassuna NA, AbdelAziz RA, Zakaria A, Abdelhakeem M. Extensively-Drug Resistant Klebsiella pneumoniae Recovered From Neonatal Sepsis Cases From a Major NICU in Egypt. Front Microbiol. 2020;11:1375. doi:10.3389/fmicb.2020.01375

7. Luo K, Tang J, Qu Y, et al. Nosocomial infection by Klebsiella pneumoniae among neonates: a molecular epidemiological study. $J$ Hosp Infect. 2021;108:174-180. doi:10.1016/j.jhin.2020.11.028

8. Vink J, Edgeworth J, Bailey SL. Acquisition of MDR-GNB in hospital settings: a systematic review and meta-analysis focusing on ESBL-E. $J$ Hosp Infect. 2020;106(3):419-428. doi:10.1016/j. jhin.2020.09.006
9. Baek EH, Kim SE, Kim S, et al. Successful control of an extendedspectrum beta-lactamase-producing Klebsiella pneumoniae ST307 outbreak in a neonatal intensive care unit. BMC Infect Dis. 2020;20 (1):166. doi:10.1186/s12879-020-4889-z

10. Awosile B, Agbaje M. Genetic Environments of Plasmid-Mediated blaCTXM-15 beta-lactamase gene in Enterobacteriaceae from Africa. Preprints. 2020;2020120144.

11. O'Connor C, Philip RK, Kelleher J, et al. The first occurrence of a CTX-M ESBL-producing Escherichia coli outbreak mediated by mother to neonate transmission in an Irish neonatal intensive care unit. BMC Infect Dis. 2017;17(1):16. doi:10.1186/s12879-016-2142-6

12. Frenk S, Rakovitsky N, Temkin E, et al. Investigation of Outbreaks of Extended-Spectrum Beta-Lactamase-Producing Klebsiella Pneumoniae in Three Neonatal Intensive Care Units Using Whole Genome Sequencing. Antibiotics. 2020;9(10):705. doi:10.3390/antibiotics9100705

13. CLSI. Performance Standards for Antimicrobial Susceptibility Testing. 30th. CLSI supplement M100. Wayne, PA: Clinical and Laboratory Standards Institute; 2020

14. Hassan H, Abdalhamid B. Molecular characterization of extendedspectrum beta-lactamase producing Enterobacteriaceae in a Saudi Arabian tertiary hospital. J Infect Dev Ctries. 2014;8(3):282-288. doi: $10.3855 /$ jidc. 3809

15. Khan MA, Lemmens N, Riera E, et al. Dominance of CTX-M-2 and CTX-M-56 among extended-spectrum beta-lactamases produced by Klebsiella pneumoniae and Escherichia coli isolated in hospitals in Paraguay. J Antimicrob Chemother. 2009;64(6):1330-1332. doi: $10.1093 / \mathrm{jac} / \mathrm{dkp} 382$

16. Tartof SY, Solberg OD, Manges AR, Riley LW. Analysis of a uropathogenic Escherichia coli clonal group by multilocus sequence typing. J Clin Microbiol. 2005;43(12):5860-5864. doi:10.1128/ JCM.43.12.5860-5864.2005

17. Diancourt L, Passet V, Verhoef J, Grimont PA, Brisse S. Multilocus sequence typing of Klebsiella pneumoniae nosocomial isolates. $J$ Clin Microbiol. 2005;43(8):4178-4182. doi:10.1128/JCM.43.8.41784182.2005

18. Kilani RA, Basamad M. Pattern of proven bacterial sepsis in a neonatal intensive care unit in Riyadh-Saudi Arabia: a 2-year analysis. J Med Liban. 2000;48(2):77-83.

19. Ohlsson A, Bailey T, Takieddine F. Changing etiology and outcome of neonatal septicemia in Riyadh, Saudi Arabia. Acta Paediatr Scand. 1986;75(4):540-544. doi:10.1111/j.1651-2227.1986.tb10246.x

20. Alqasim A. Extraintestinal pathogenic Escherichia coli in Saudi Arabia: a review of antimicrobial resistance and molecular epidemiology. Tropical J Pharmaceutical Res. 2020;19(2):447-453. doi:10.4314/tjpr.v19i2.30

21. Alqasim A, Jaffal AA, Almutairi N, Arshad M, Alyousef AA. Isolation, phenotypic and genotypic characterization of Escherichia coli from the bloodstream samples in Riyadh, Saudi Arabia. $J$ King Saud Univ. 2020;32(2):1464-1469. doi:10.1016/j.jksus.2019.11.043

22. Jafari A, Falahatkar S, Delpasand K, Sabati H, Sedigh Ebrahim-Saraie H. Emergence of Escherichia coli ST131 Causing Urinary Tract Infection in Western Asia: a Systematic Review and Meta-Analysis. Microb Drug Resist. 2020;26(11):1357-1364. doi:10.1089/mdr.2019.0312

23. Kondratyeva K, Salmon-Divon M, Navon-Venezia S. Meta-analysis of Pandemic Escherichia coli ST131 Plasmidome Proves Restricted Plasmid-clade Associations. Sci Rep. 2020;10(1):36. doi:10.1038/ s41598-019-56763-7

24. Mshana SE, Hain T, Domann E, et al. Predominance of Klebsiella pneumoniaeST14 carrying CTX-M-15 causing neonatal sepsis in Tanzania. BMC Infect Dis. 2013;13(1):466. doi:10.1186/1471-233413-466

25. Oteo J, Cuevas O, López-Rodríguez I, et al. Emergence of CTX-M15-producing Klebsiella pneumoniae of multilocus sequence types 1, $11,14,17,20,35$ and 36 as pathogens and colonizers in new-borns and adults. $J$ Antimicrob Chemother. 2009;64(3):524-528. doi:10.1093/jac/dkp211 
26. Tawfik AF, Alswailem AM, Shibl AM, Al-Agamy MH. Prevalence and genetic characteristics of TEM, SHV, and CTX-M in clinical Klebsiella pneumoniae isolates from Saudi Arabia. Microb Drug Resist. 2011;17(3):383-388. doi:10.1089/mdr.2011.0011

27. Shibl AM, Al-Agamy MH, Khubnani H, Senok AC, Tawfik AF, Livermore DM. High prevalence of acquired quinolone-resistance genes among Enterobacteriaceae from Saudi Arabia with CTX-M15 ß-lactamase. Diagn Microbiol Infect Dis. 2012;73(4):350-353. doi:10.1016/j.diagmicrobio.2012.04.005

28. Al-Agamy MHM, Shibl AM, Tawfik AF, Elbannai AR. Klebsiella pneumoniae Producing CTX-M-15 Genes from Neonatal Intensive Care Unit in Saudi Arabia. Res J Microbiol. 2009;4(7):278-285. doi:10.3923/jm.2009.278.285

29. Peirano G, Pitout JDD. Extended-Spectrum $\beta$-Lactamase-Producing Enterobacteriaceae: update on Molecular Epidemiology and Treatment Options. Drugs. 2019;79(14):1529-1541. doi:10.1007/ s40265-019-01180-3

30. Bindayana KM, Joji RM, Ezzat H, Jahrami HA. Genes conferring antimicrobial-resistance among Klebsiella pneumoniae in the Arabian Gulf countries: a systemic review and meta-analysis. Asian J Pharm Clin Res. 2021;14(4):90-99. doi:10.22159/ajpcr.2021.v14i4.40841
31. Balkhy HH, El-Saed A, AlShehri A, et al. Antimicrobial consumption in three pediatric and neonatal intensive care units in Saudi Arabia: 33-month surveillance study. Ann Clin Microbiol Antimicrob. 2019;18(1):20. doi:10.1186/s12941-019-0320-2

32. Mahfouz AA, Al-Azraqi TA, Abbag FI, Al-Gamal MN, Seef S, Bello CS. Nosocomial infections in a neonatal intensive care unit in southwestern Saudi Arabia. East Mediterr Health J. 2010;16(1):40-44. doi:10.26719/2010.16.1.40

33. Al-Matary A, Heena H, AlSarheed AS, et al. Characteristics of neonatal Sepsis at a tertiary care hospital in Saudi Arabia. $J$ Infect Public Health. 2019;12(5):666-672. doi:10.1016/j.jiph.2019.03.007

34. Babini GS, Livermore DM. Are SHV beta-lactamases universal in Klebsiella pneumoniae? Antimicrob Agents Chemother. 2000;44 (8):2230. doi:10.1128/AAC.44.8.2230-2230.2000
Infection and Drug Resistance

\section{Publish your work in this journal}

Infection and Drug Resistance is an international, peer-reviewed openaccess journal that focuses on the optimal treatment of infection (bacterial, fungal and viral) and the development and institution of preventive strategies to minimize the development and spread of resistance. The journal is specifically concerned with the epidemiology of
Dovepress

antibiotic resistance and the mechanisms of resistance development and diffusion in both hospitals and the community. The manuscript management system is completely online and includes a very quick and fair peerreview system, which is all easy to use. Visit http://www.dovepress.com/ testimonials.php to read real quotes from published authors. 